\title{
ON APPROXIMATION CONSTANTS FOR LIOUVILLE NUMBERS
}

\author{
JOHANNES SCHLEISCHITZ
}

University of Natural Resources and Life Sciences, Austria

\begin{abstract}
We investigate some Diophantine approximation constants related to the simultaneous approximation of $\left(\zeta, \zeta^{2}, \ldots, \zeta^{k}\right)$ for Liouville numbers $\zeta$. For a certain class of Liouville numbers including the famous representative $\sum_{n>1} 10^{-n !}$ and numbers in the Cantor set, we explicitly determine all approximation constants simultaneously for all $k \geq 1$.
\end{abstract}

\section{Definition and BAsic Properties of $\lambda_{k, j}, \widehat{\lambda}_{k, j}$}

1.1. One dimensional approximation. We begin with the definition of the irrationality exponent. For a real number $\zeta$ it is denoted by $\mu(\zeta)$ and defined as the supremum of $\eta \in \mathbb{R}$ for which

$$
\left|\zeta-\frac{y}{x}\right| \leq x^{-\eta}
$$

has infinitely many solutions in rational numbers $y / x$. It follows from Dirichlet's Theorem, [29, Corollary 2], that $\mu(\zeta) \geq 2$ for all $\zeta \in \mathbb{R} \backslash \mathbb{Q}$, whereas obviously $\mu(\zeta)=1$ for $\zeta \in \mathbb{Q}$. Capelli showed that actually $\mu(\zeta)=2$ for Lebesgue almost all $\zeta \in \mathbb{R}$, which was generalized first by Khintchine and later by Beresnevich, Dickinson and Velani ([1]). Roth's Theorem ([13]) asserts $\mu(\zeta)=2$ for all algebraic irrational real numbers $\zeta$. Real numbers $\zeta$ with $\mu(\zeta)=\infty$ are called Liouville numbers. Liouville's first example of a transcendental number

$$
L:=\sum_{n \geq 1} 10^{-n !}=10^{-1}+10^{-2}+10^{-6}+10^{-24}+\cdots
$$

2010 Mathematics Subject Classification. 11J13, 11J25, 11J82.

Key words and phrases. Geometry of numbers, successive minima, Liouville numbers, Diophantine approximation.

Supported by the Austrian Science Fund FWF grant P24828. 
is easily verified to be a Liouville number.

1.2. Simultaneous approximation: The $\mathbb{Q}$-linear independent case. Generalizing this concept for simultaneous approximation of $\underline{\zeta}=\left(\zeta_{1}, \ldots, \zeta_{k}\right) \in \mathbb{R}^{k}$, for $1 \leq j \leq k+1$ define $\lambda_{k, j}(\underline{\zeta})$ resp. $\widehat{\lambda}_{k, j}(\underline{\zeta})$ the supremum of $\eta \in \mathbb{R}$ for which the system

$$
\begin{aligned}
|x| & \leq X \\
\max _{1 \leq j \leq k}\left|\zeta_{j} x-y_{j}\right| & \leq X^{-\eta}
\end{aligned}
$$

has (at least) $j$ linearly independent solutions $\left(x, y_{1}, y_{2}, \ldots, y_{k}\right) \in \mathbb{Z}^{k+1}$ for arbitrarily large real $X$ resp. for all $X \geq X_{0}$. Note that for $k=1, \zeta=\zeta \notin \mathbb{Q}$ the identity $\lambda_{1,1}(\zeta)+1=\mu(\zeta)$ holds. Clearly, for all $\underline{\zeta} \in \mathbb{R}^{k}$ the inequalities

$$
\begin{aligned}
& \lambda_{k, 1}(\underline{\zeta}) \geq \lambda_{k, 2}(\underline{\zeta}) \geq \cdots \geq \lambda_{k, k+1}(\underline{\zeta}) \geq 0, \\
& \widehat{\lambda}_{k, 1}(\underline{\zeta}) \geq \widehat{\lambda}_{k, 2}(\underline{\zeta}) \geq \cdots \geq \widehat{\lambda}_{k, k+1}(\underline{\zeta}) \geq 0,
\end{aligned}
$$

and

$$
\lambda_{k, j}(\underline{\zeta}) \geq \widehat{\lambda}_{k, j}(\underline{\zeta}), \quad 1 \leq j \leq k+1
$$

hold. Furthermore, a generalization of Dirichlet's Theorem asserts

$$
\lambda_{k, 1}(\underline{\zeta}) \geq \widehat{\lambda}_{k, 1}(\underline{\zeta}) \geq \frac{1}{k}
$$

for all $\zeta \in \mathbb{R}^{k}$. This can be found in [29, page 19, Chapter 2.4]. Again, there is actually equality in both inequalities in (1.7) for Lebesgue almost all $\zeta \in \mathbb{R}^{k}$, such as for all algebraic $\underline{\zeta} \in \overline{\mathbb{Q}}^{k}$ for which $\left\{1, \zeta_{1}, \ldots, \zeta_{k}\right\}$ is $\mathbb{Q}$-linearly independent. The first assertion was established by Khintchine, the latter is a consequence of Schmidt's Subspace Theorem ([23]). Define

$$
\begin{aligned}
& \chi_{k, 1}=\chi_{k, 2}=\frac{1}{k}, \quad \chi_{k, 3}=\chi_{k, 4}=\cdots=\chi_{k, k+1}=0, \\
& \phi_{k, 1}=\frac{1}{k}, \quad \phi_{k, 2}=\phi_{k, 3}=\cdots=\phi_{k, k+1}=0 .
\end{aligned}
$$

For $\underline{\zeta} \in \mathbb{R}^{k}$ that satisfies the $\mathbb{Q}$-linear independence property above, see [19, (14)-(19)] (where $\omega_{j}$ corresponds to the present $\lambda_{k, j}(\underline{\zeta})$ ) translate into

$$
\begin{array}{rlrl}
\chi_{k, j} \leq \lambda_{k, j}(\underline{\zeta}) & \leq \frac{1}{j-1}, & & 1 \leq j \leq k+1, \\
\phi_{k, j} \leq \widehat{\lambda}_{k, j}(\underline{\zeta}) & \leq \frac{1}{j}, & & 1 \leq j \leq k, \\
\phi_{k, k+1} \leq \widehat{\lambda}_{k, k+1}(\underline{\zeta}) & \leq \frac{1}{k} . &
\end{array}
$$

Here we have put $1 / 0=\infty$ and we agree on this such as $1 / \infty=0$ anywhere it appears. Individually, these bounds are best possible, as deduced in [19, Corollary 9]. In fact, the linear independence property is required only in 
(1.10). Several other restrictions concerning the joint spectrum of the quantities $\lambda_{k, j}$ resp. $\widehat{\lambda}_{k, j}$ are known, mostly inferred via Minkowski's second lattice point Theorem ([12], see also $[24,25])$.

We want to make some notational remarks. If we would demand the maximum in (1.4) to be strictly larger than 0 , as for the related constants $\lambda_{k}(\zeta)$ defined in [4], the identity $\lambda_{1,1}(\zeta)+1=\mu(\zeta)$ would hold for $\zeta \in \mathbb{Q}$ too. Moreover, the condition $\lambda_{1,1}(\zeta)=\infty$ would imply that $\zeta$ is a Liouville number without the additional condition that $\zeta$ is irrational. However, inconvenient inconsistencies with results from Schmidt and Summerer $([24,25])$ would arise, who do not require the additional condition. We should mention that their notation, used for example in [24], for $\lambda_{k, 1}(\underline{\zeta}) \operatorname{resp} . \widehat{\lambda}_{k, 1}(\underline{\zeta})$ is $\omega \operatorname{resp} . \widehat{\omega}$, where the vector $\zeta$ is considered fixed and its dimension is omitted in the notation. There is no actual standard notation for the generalized constants where $j \geq 2$, in [19] the notation $\omega_{j}$ resp. $\widehat{\omega}_{j}$ is used. We use the present notation because it allows convenient relation to the successive power case we will introduce in Section 1.3.

1.3. Simultaneous approximation: The successive power case. An interesting heavily studied special case is that $\zeta$ as in Section 1.2 consists of the first successive powers of a real number $\zeta$, i.e. $\zeta=\left(\zeta, \zeta^{2}, \ldots, \zeta^{k}\right)$. We will mostly deal with this case in the sequel, so for simplicity for any pair of positive integers $k, j$ with $1 \leq j \leq k+1$ we will write

$$
\lambda_{k, j}(\zeta):=\lambda_{k, j}\left(\zeta, \zeta^{2}, \ldots, \zeta^{k}\right), \quad \widehat{\lambda}_{k, j}(\zeta):=\widehat{\lambda}_{k, j}\left(\zeta, \zeta^{2}, \ldots, \zeta^{k}\right) .
$$

Throughout, we assume the $\mathbb{Q}$-linear independence condition from the previous Section 1.2 to hold, which has the natural interpretation that $\zeta$ is not algebraic of degree $\leq k$. We remark that again our definition of $\lambda_{k, 1}(\zeta)$ slightly differs from the quantity $\lambda_{k}(\zeta)$ defined in [4] due to not asking for the maximum in (1.4) to be positive, however the inconsistency appears for rational $\zeta$ only.

Lebesgue almost all $\zeta$ satisfy $\lambda_{k, j}(\zeta)=\widehat{\lambda}_{k, j}(\zeta)=1 / k$ for all pairs $j, k$ with $1 \leq j \leq k+1$. This was established by Sprindẑuk ([27]) for $j=1$, combination with Minkowski's second lattice point Theorem readily yields the general assertion. Generalizations of this metric result for $\underline{\zeta}=\left(\zeta_{1}, \zeta_{2}, \ldots, \zeta_{k}\right)$ belonging to a wider class of algebraic curves in $\mathbb{R}^{k}$ can be found in [8].

Clearly all inequalities and bounds established in Section 1.2 hold for the special case of successive powers as well. However, the bounds (1.9)(1.11) are in general not optimal, particularly those related to the uniform approximation constants (with the "hat") differ significantly. Indeed, the upper bound

$$
\widehat{\lambda}_{k, 1}(\zeta) \leq\left\lceil\frac{k}{2}\right\rceil^{-1}
$$


valid for all $\zeta$ not algebraic of degree $\leq\lceil k / 2\rceil$ is due to Davenport, Schmidt and Laurent $([9])$. Here as usual $\lceil\alpha\rceil$ denotes the smallest integer greater or equal to $\alpha \in \mathbb{R}$. Indeed $\lceil k / 2\rceil^{-1}$ sharpens the bound 1 for $\widehat{\lambda}_{k, 1}(\zeta)$ arising from (1.10) vastly, and (1.6) further implies sharper restrictions on the spectrum of $\widehat{\lambda}_{k, j}(\zeta)$ at least for some indices $2 \leq j \leq k+1$ too.

The spectrum of $\lambda_{k, j}(\zeta)$ among all real numbers $\zeta$ in dependence of $k$ has been studied as well, mostly for $j=1$. Bugeaud proved in [3, Theorem 2] that the spectrum of $\lambda_{k, 1}$ contains $[1, \infty]$, a generalization due to the author is given in [22]. The natural conjecture already quoted in [3] is the following.

Conjecture 1.1. Let $k$ be a positive integer. The spectrum of $\lambda_{k, 1}(\zeta)$ among all real numbers $\zeta$ not algebraic of degree $\leq k$ is $[1 / k, \infty]$.

Apart from $k=1$ this is only known for $k=2$ thanks to Beresnevich, Dickinson, Vaughan and Velani $([2,28])$. For further problems and results in this manner (see also [3]). We will return to the spectrum of $\lambda_{k, j}(\zeta), \widehat{\lambda}_{k, j}(\zeta)$ for fixed $k$ in Section 2 .

It follows from their definition that for fixed $\zeta$ and $j \geq 1$, the quantities $\lambda_{k, j}(\zeta), \widehat{\lambda}_{k, j}(\zeta)$ are non-increasing as $k$ increases, i.e. (with $\lambda_{0,1}(\zeta):=$ $\left.\infty, \widehat{\lambda}_{0,1}(\zeta):=\infty\right)$

$$
\begin{aligned}
& \lambda_{j-1, j}(\zeta) \geq \lambda_{j, j}(\zeta) \geq \lambda_{j+1, j}(\zeta) \geq \cdots, \quad j \geq 1, \\
& \widehat{\lambda}_{j-1, j}(\zeta) \geq \widehat{\lambda}_{j, j}(\zeta) \geq \widehat{\lambda}_{j+1, j}(\zeta) \geq \cdots, \quad j \geq 1 .
\end{aligned}
$$

For $j=1$, [3, Lemma 1] by Bugeaud states a result somehow reverse to (1.13), which in our notation says the following.

TheOREM 1.2 (Bugeaud). For any positive integers $k$ and $n$, and any transcendental real number $\zeta$ we have

$$
\lambda_{k n, 1}(\zeta) \geq \frac{\lambda_{n, 1}(\zeta)-k+1}{k} .
$$

There is actually equality in case of $\lambda_{k n, 1}(\zeta)>1$, as established by the author ([21]). If we put $n=1$ in Theorem 1.2 and let $\zeta$ be a Liouville number, we obtain [3, Corollary 2].

Corollary 1.3 (Bugeaud). Let $\zeta$ be an irrational real number. We have $\lambda_{k, 1}(\zeta)=\infty$ for all positive integers $k$ if and only if $\lambda_{1,1}(\zeta)=\infty$, i.e. $\zeta$ is a Liouville number.

We shall utilize Corollary 1.3 in the proof of Theorem 3.1.

\section{The speCtrum of $\lambda_{k, j}(\zeta), \widehat{\lambda}_{k, j}(\zeta)$ : KNOWN FACTS}

In this section we quote facts on the individual such as the joint spectrum of the quantities $\lambda_{k, j}(\zeta), \widehat{\lambda}_{k, j}(\zeta)$. Let us restrict to $j=1$ first. The restriction (1.12) shows that for not too small values of $\lambda_{k, 1}(\zeta)$, there are far 
more stringent restrictions for the joint spectrum of $\left(\lambda_{k, 1}(\zeta), \widehat{\lambda}_{k, 1}(\zeta)\right)$ than (1.7). Another result that affirms this arises from the following observation, which is basically [21, Theorem 1.12 ] due to the author. Due to the deviant definitions of the present $\lambda_{k, 1}$ and $\lambda_{k}$ in [4] for $\zeta \in \mathbb{Q}$, we need to restrict to irrational $\zeta$ in the present formulation. The same will apply to Corollary 2.2 .

TheOrem 2.1 (S, 2014). Let $\zeta \in \mathbb{R} \backslash \mathbb{Q}$. For any positive integer $k$, we have

$$
\widehat{\lambda}_{k, 1}(\zeta) \leq \max \left\{\frac{1}{\lambda_{1,1}(\zeta)}, \frac{1}{k}\right\}
$$

We are again particularly interested in the consequences for Liouville numbers. The following corollary is basically contained in [21, Corollary 5.2].

Corollary 2.2. Let $k$ be a positive integer. Let $\zeta \in \mathbb{R} \backslash \mathbb{Q}$ such that $\lambda_{1,1}(\zeta) \geq k$, which is in particular true if $\lambda_{k, 1}(\zeta)>1$. Then

$$
\widehat{\lambda}_{k, 1}(\zeta)=\frac{1}{k} \text {. }
$$

In particular, if $\zeta$ is a Liouville number, then $\widehat{\lambda}_{k, 1}(\zeta)=1 / k$ simultaneously for all $k$.

Proof. Combining (1.7) and Theorem 2.1 and observation subsequent to Theorem 1.2, for $\lambda_{k, 1}(\zeta)>1$ we obtain $\lambda_{1,1}(\zeta)=k \lambda_{k, 1}(\zeta)+k-1>2 k-1 \geq k$.

We should mention that for $k=2$, the maximum value of $\widehat{\lambda}_{2,1}(\zeta)$ among all $\zeta$ not algebraic of degree $\leq 2$ is known. It is attained for so called extremal numbers $\zeta$, which satisfy $\lambda_{2,1}(\zeta)=1, \widehat{\lambda}_{2,1}(\zeta)=\gamma:=(\sqrt{5}-1) / 2$, see [14]. Besides, extremal numbers provide explicit vectors $\underline{\zeta}=\left(\zeta, \zeta^{2}\right)$ where equality holds in inequality $[25,(1.21)]$ for $k=2$, which is in our notation

$$
\lambda_{k, 1} \underline{(\zeta)} \geq \frac{\widehat{\lambda}_{k, 1}(\underline{\zeta})^{2}+(k-2) \widehat{\lambda}_{k, 1}(\underline{\zeta})}{(k-1)\left(1-\widehat{\lambda}_{k, 1}(\underline{\zeta})\right)} .
$$

Note that (2.1) is valid for any $k \geq 2$ and $\underline{\zeta} \in \mathbb{R}^{k}$ which is $\mathbb{Q}$-linearly independent together with 1 . More generally, an extremal number induces a certain geometric pattern which Summerer and Schmidt call a regular graph, see [26]. See also their papers $[24,25]$ such as Roy's recent papers [16-18] for the (joint) spectrum of $\lambda_{k, j}(\underline{\zeta}), \widehat{\lambda}_{k, j}(\underline{\zeta})$ in the $\mathbb{Q}$-linearly independent case of Section 1.2.

Observe that for $k=2$ the estimate (1.12) would only yield the trivial upper bound 1 from (1.10), that only requires the linear independence assumption on $\zeta$, which is significantly larger than $\gamma \approx 0.6180$. For $k=3$, the bound $1 / 2$ from (1.12) was improved as well by Roy ([15]), however the best value remains unknown. For $k \geq 4$, no improvements concerning the spectrum of the constants $\lambda_{k, 1}(\zeta), \widehat{\lambda}_{k, 1}(\zeta)$ in this spirit have been made. 
The quantities $\lambda_{k, j}(\zeta), \widehat{\lambda}_{k, j}(\zeta)$ for $2 \leq j \leq k+1$ remain very poorly studied. The remainder of this paper is devoted to this question in the special case of Liouville numbers $\zeta$. It will turn out in Theorem 3.1 that for Liouville numbers all uniform constants $\widehat{\lambda}_{k, j}(\zeta)$ can be explicitly determined. Moreover, Theorem 3.2 will suggest Conjecture 5.6 on the (individual) spectrum of each $\lambda_{k, j}(\zeta)$ for $\zeta$ not algebraic of degree $\leq k$.

\section{Formulation of the MAin RESUlts}

3.1. The general case. The concern of the first theorem is to determine/bound the classic approximation constants for arbitrary Liouville numbers.

THEOREM 3.1. Let $\zeta$ be a Liouville number. For any positive integer $k$, we have

$$
\begin{aligned}
\lambda_{k, 1}(\zeta) & =\infty, \\
\frac{1}{k} \leq \lambda_{k, j}(\zeta) & \leq \frac{1}{j-1}, \quad 2 \leq j \leq k+1, \\
\widehat{\lambda}_{k, 1}(\zeta) & =\frac{1}{k}, \\
\widehat{\lambda}_{k, j}(\zeta) & =0,
\end{aligned}
$$

3.2. A special class of Liouville numbers. We provide a class of Liouville numbers $\zeta$, for which all approximation constants $\lambda_{k, j}(\zeta), \widehat{\lambda}_{k, j}(\zeta)$ can be determined explicitly simultaneously for all $k$. The weakest assumptions on $\zeta$ for our methods to work will be those in Theorem 3.2. Various specializations will be given in Section 5.2.

Theorem 3.2. Let $k$ be a positive integer. Further let $q_{1}, q_{2}, \ldots$ be positive integers such that $q_{l} \mid q_{l+1}$ and $q_{l}<q_{l+1}$ for all $l \geq 1$. Suppose that for a positive integer $k$ and all $C>0$ there exists $n=n(C)$ such that

$$
\frac{\log q_{n+1}}{\log q_{n}}>C, \quad \frac{\log q_{n+2}}{\log q_{n+1}}>k+1 .
$$

Further set

$$
\zeta=\sum_{l \geq 1} \frac{1}{q_{l}}
$$

Then we have

$$
\begin{aligned}
\lambda_{k, j}(\zeta) & =\frac{1}{j-1}, \quad 1 \leq j \leq k+1 \\
\widehat{\lambda}_{k, 1}(\zeta) & =\frac{1}{k}, \\
\widehat{\lambda}_{k, j}(\zeta) & =0, \quad 2 \leq j \leq k+1 .
\end{aligned}
$$


We will prove both Theorems 3.1 and 3.2 and give some remarks in Section 5.1. Further, we will discuss some consequences in Section 5.2.

\section{Preparatory Results for the proofs}

Parts of the proofs of the main new results in Section 3 can be generalized, so we will state the more general versions in form of Lemmas 4.1 and 4.2. The proofs of those lemmas are basically a consequence of Minkowski's second lattice point Theorem, hidden in the results from $[24,25]$ we utilize.

We introduce the functions $\psi_{k, j}$ and the derived quantities $\underline{\psi}_{k, j}, \bar{\psi}_{k, j}$ defined in [24] with a slightly different notation (subindex $k$ omitted). For fixed $\zeta=\left(\zeta_{1}, \ldots, \zeta_{k}\right) \in \mathbb{R}^{k}$ and a real parameter $Q>1$, for $1 \leq j \leq k+1$ define $\bar{\psi}_{k, j}(Q)$ as the supremum of all values of $\nu \in \mathbb{R}$ such that the system

$$
\begin{aligned}
|x| & \leq Q^{1+\nu}, \\
\max _{1 \leq j \leq k}\left|\zeta_{j} x-y_{j}\right| & \leq Q^{-\frac{1}{k}+\nu}
\end{aligned}
$$

has (at least) $j$ linearly independent solutions $\left(x, y_{1}, \ldots, y_{k}\right) \in \mathbb{Z}^{k+1}$. Further, let

$$
\underline{\psi}_{k, j}=\liminf _{Q \rightarrow \infty} \psi_{k, j}(Q), \quad \bar{\psi}_{k, j}=\limsup _{Q \rightarrow \infty} \psi_{k, j}(Q) .
$$

The quantities obviously have the properties $\underline{\psi}_{k, j} \leq \bar{\psi}_{k, j}$ and

$$
\begin{aligned}
& -1 \leq \underline{\psi}_{k, 1} \leq \underline{\psi}_{k, 2} \leq \cdots \leq \underline{\psi}_{k, k+1} \leq 1 / k, \\
& -1 \leq \bar{\psi}_{k, 1} \leq \bar{\psi}_{k, 2} \leq \cdots \leq \bar{\psi}_{k, k+1} \leq 1 / k .
\end{aligned}
$$

Minkowski's first lattice point Theorem (or Dirichlet's Theorem) further implies

$$
\bar{\psi}_{k, 1} \leq 0 .
$$

Moreover, the generalization $[19,(13)]$ of $[24,(1.9)]$ for arbitrary $j$, shows that $\underline{\psi}_{k, j}$ and $\lambda_{k, j}=\lambda_{k, j}(\underline{\zeta})$ such as $\bar{\psi}_{k, j}$ and $\hat{\lambda}_{k, j}=\widehat{\lambda}_{k, j}(\underline{\zeta})$ are closely connected. In our notation the correspondence is given by

(4.4) $\left(1+\lambda_{k, j}\right)\left(1+\underline{\psi}_{k, j}\right)=\left(1+\widehat{\lambda}_{k, j}\right)\left(1+\bar{\psi}_{k, j}\right)=\frac{k+1}{k}, \quad 1 \leq j \leq k+1$.

We will use (4.4) frequently in the proof of the following Lemmas 4.1, 4.2. Moreover, we will utilize [25, (1.10)], which in the notation of the present Section 4 states

$$
\begin{array}{ll}
j \underline{\psi}_{k, 1}+(k+1-j) \bar{\psi}_{k, j+1} \leq 0, & 0 \leq j \leq k, \\
j \bar{\psi}_{k, 1}+(k+1-j) \underline{\psi}_{k, j+1} \leq 0, & 0 \leq j \leq k .
\end{array}
$$

Further we need

$$
\bar{\psi}_{k, 1}+k \underline{\psi}_{k, k+1} \geq 0
$$


which is the right estimate in $[25,(1.13)]$. Finally we need inequality $[24$, (1.6a)] for $i=1$, which is in the present notation

$$
\underline{\psi}_{k, 1}+\bar{\psi}_{k, 2}+\bar{\psi}_{k, 3}+\cdots+\bar{\psi}_{k, k+1} \geq 0 .
$$

Before we state and prove the lemmas, we should add that the functions $\psi_{k, j}(Q)$ and thus the derived values $\underline{\psi}_{k, j}, \bar{\psi}_{k, j}$ have a natural geometric interpretation as successive minima of special convex bodies with respect to a lattice. However, we will not explicitly use the geometric view for our approaches and refer to [24] for details.

LEMmA 4.1. For any positive integer $k$ and any $\underline{\zeta} \in \mathbb{R}^{k}$ we have the equivalence

$$
\lambda_{k, 1}(\underline{\zeta})=\infty \quad \Longleftrightarrow \quad \widehat{\lambda}_{k, 2}(\underline{\zeta})=\widehat{\lambda}_{k, 3}(\underline{\zeta})=\cdots=\widehat{\lambda}_{k, k+1}(\underline{\zeta})=0 .
$$

PROOF. Actually, the author basically deduces the left to right implication of (4.9) in the last part of the proof of [19, Theorem 1] with the aid of the quantities $\underline{\psi}_{k, j}, \bar{\psi}_{k, j}$. We want to prove it again rigorously, however. With (4.4) one can reinterpret $\lambda_{k, 1}(\underline{\zeta})=\infty$ as $\underline{\psi}_{k, 1}=-1$. Together with inequality (4.8) and noting $\bar{\psi}_{j} \leq 1 / k$ for all $1 \leq j \leq k+1$ by (4.2), we conclude $\bar{\psi}_{k, 2}=\cdots=\bar{\psi}_{k, k+1}=1 / k$. Again reinterpreting this using (4.4), the right hand side of (4.9) follows. We prove the other direction in (4.9). First note that (4.4) with $j=2$ and the right hand side of (4.9) yields $\bar{\psi}_{k, 2}=1 / k$. Applying (4.5) for $j=1$ further gives $\underline{\psi}_{k, 1}+1=\underline{\psi}_{k, 1}+k \bar{\psi}_{k, 2} \leq 0$. Hence (4.1) implies $\underline{\psi}_{k, 1}=-1$, and again with (4.4) for $j=1$ finally $\lambda_{k, 1}(\underline{\zeta})=\infty$.

口

LEMMA 4.2. Let $k$ be a positive integer and $\underline{\zeta} \in \mathbb{R}^{k}$ arbitrary. Then

$$
\widehat{\lambda}_{k, 1}(\underline{\zeta})=\frac{1}{k} \quad \Longleftrightarrow \quad \lambda_{k, 2}(\underline{\zeta}) \geq \lambda_{k, 3}(\underline{\zeta}) \geq \cdots \geq \lambda_{k, k+1}(\underline{\zeta})=\frac{1}{k} .
$$

Proof. First note that by (1.5), it remains to show the equivalence of the left hand side to the last equality on the right hand side. The implication left to right of the lemma is a consequence of (4.6). By (4.4) we know $\widehat{\lambda}_{k, 1}(\underline{\zeta})=$ $1 / k$ is equivalent to $\bar{\psi}_{k, 1}=0$, hence (4.6) implies $\underline{\psi}_{k, j} \leq 0$ for all $1 \leq j \leq \bar{k}+1$, and again with (4.4) we conclude $\lambda_{k, k+1}(\underline{\zeta}) \geq 1 / k$. The reverse inequality $\lambda_{k, k+1}(\underline{\zeta}) \leq 1 / k$ is by $(4.4)$ equivalent to $\underline{\psi}_{k, k+1} \geq 0$, but (4.7) and (4.3) indeed imply

$$
\underline{\psi}_{k, k+1} \geq-\frac{1}{k} \bar{\psi}_{k, 1} \geq 0 .
$$

We turn to the implication right to left. Suppose $\lambda_{k, k+1}(\underline{\zeta})=1 / k$, which by (4.4) is equivalent to $\underline{\psi}_{k, k+1}=0$. On the other hand, by virtue of (4.4), 
the assertion $\widehat{\lambda}_{k, 1}(\underline{\zeta})=1 / k$ is equivalent to $\bar{\psi}_{k, 1}=0$. However, this is a consequence of $\underline{\psi}_{k, k+1}=0,(4.3)$ and (4.10).

\section{Proofs of Theorems 3.1, 3.2 and consequences}

5.1. Proofs. Theorem 3.1 is readily inferred by combining the results of Section 2 with the results we have established in Section 4.

Proof of Theorem 3.1. Corollary 1.3 yields (3.1) and Lemma $4.1 \mathrm{im}-$ plies (3.4). The upper bounds in (3.2) are due to (1.9), since the $\mathbb{Q}$-linear independence condition is certainly satisfied as Liouville numbers are transcendental by Roth's Theorem, see Section 1.1. The lower bounds in (3.2) are due to Lemma 4.2. Finally Corollary 2.2 gives (3.3).

We turn to the more technical proof of Theorem 3.2.

Proof of Theorem 3.2. The conditions on the sequence $\left(q_{l}\right)_{l \geq 1}$ imply $q_{l+1} \geq 2 q_{l}$ and thus the sum converges and $\zeta$ as in the theorem is well-defined. It is further easily checked by means of (3.5) that $\zeta$ is a Liouville number. In view of Theorem 3.1, it suffices to prove

$$
\lambda_{k, j}(\zeta) \geq \frac{1}{j-1}, \quad 2 \leq j \leq k+1 .
$$

Suppose that for each $C>0$ there exists a large index $n=n(C)$ for which (3.5) is satisfied. We consider the pair $C, n$ fixed in the sequel. Define the integers

$$
U=q_{n}, \quad V=q_{n+1}, \quad A=q_{n} \sum_{l=1}^{n} \frac{1}{q_{l}} .
$$

For each pair $(j, m) \in\{1,2, \ldots, k+1\}^{2}$ denote $E_{j, m}$ the closest integer to $U^{k} V^{j-1} \zeta^{m-1}$ and let

$$
E_{j}=\left(E_{j, 1}, \ldots, E_{j, k+1}\right), \quad 1 \leq j \leq k+1 .
$$

In view of the condition $\log q_{n+2} / \log q_{n+1}>k+1$, we see that $\zeta$ is very close to $U^{-1} A+V^{-1}$, more precisely

$$
\left|\zeta-\left(U^{-1} A+V^{-1}\right)\right| \ll V^{-k-1} .
$$

From (5.2) it follows that actually

$$
\left|\zeta^{m}-\left(U^{-1} A+V^{-1}\right)^{m}\right| \ll V^{-k-1}, \quad 1 \leq m \leq k .
$$

Hence, for any pair $(j, m) \in\{1,2, \ldots, k+1\}^{2}$, one checks that $E_{j, m}$ is also the closest integer to $U^{k} V^{j-1}\left(U^{-1} A+V^{-1}\right)^{m-1}$. This further implies

$$
E_{j, m}=U^{k} V^{j-1} \sum_{i=0}^{\min \{m-1, j-1\}}\left(\begin{array}{c}
m-1 \\
i
\end{array}\right)\left(\frac{A}{U}\right)^{m-1-i}\left(\frac{1}{V}\right)^{i},
$$


as the right hand side is an integer and the omitted terms in the expansion of $U^{k} V^{j-1}\left(U^{-1} A+V^{-1}\right)^{m-1}$ sum up to a very small number, clearly smaller than $1 / 2$. More precisely, from the definition of $E_{j, m}$ and (5.4), we deduce

$$
\left|E_{j, m}-U^{k} V^{j-1} \zeta^{m-1}\right| \ll U^{k} V^{j-1} V^{-j}=U^{k} V^{-1}, \quad 1 \leq j, m \leq k+1 .
$$

Observe that $E_{j, 1}=U^{k} V^{j-1} \geq V^{j-1}$ and moreover (3.5) implies $U^{k} V^{-1} \ll$ $V^{k / C-1}$. Hence (5.5) further yields

$$
\max _{1 \leq i \leq k}-\frac{\log \left\|E_{j, 1} \zeta^{i}\right\|}{\log E_{j, 1}} \geq \frac{1-\frac{k}{C}+\frac{D}{\log V}}{j-1}=: H_{j, C},
$$

with a constant $D=D(k, \zeta)$ independent from $C, n$. Moreover, since

$$
E_{1,1}<E_{2,1}<\cdots<E_{k+1,1}
$$

for any $1 \leq j \leq k+1$ the first coordinate of any of the first $j$ integer vectors $\left\{E_{1}, E_{2}, \ldots, E_{j}\right\}$ is bounded above by $E_{j, 1}$. We conclude from (5.6) that the system (1.3),(1.4) with respect to this value $X:=E_{j, 1}$ and the $j$ vectors $\left(x, y_{1}, \ldots, y_{k}\right):=E_{i}$ for $1 \leq i \leq j$, will be satisfied for a value $\eta \geq H_{j, C}$. Now observe that $H_{j, C}$ will be arbitrarily close to $1 /(j-1)$ if we let $C \rightarrow \infty$, since then both $k / C$ and $D / \log V$ tend to 0 .

It remains to prove the linear independence of $E_{1}, E_{2}, \ldots, E_{k+1}$ to deduce (5.1). This is equivalent to the regularity of the matrix $\left(E_{j, m}\right)_{1 \leq j, m \leq k+1}$. However, by virtue of $(5.4)$ the reduction of $\left(E_{j, m}\right)_{1 \leq j, m \leq k+1}$ modulo $V$ leads to an upper triangular matrix with $U^{k}$ everywhere on the diagonal, consequently its determinant in $\mathbb{Z} / V \mathbb{Z}$ equals $U^{k(k+1)}$. Provided that $C>k(k+1)$ we have $0<U^{k(k+1)}<V$ such that this determinant does not vanish, so clearly the determinant of $\left(E_{j, m}\right)_{1 \leq j, m \leq k+1}$ is non-zero as well.

REMARK 5.1. For $j>1$ the quantities $\lambda_{k, j}(\zeta)$ need not equal the upper bound $1 /(j-1)$ for any Liouville number $\zeta$ (not of the form as in Theorem 3.2). This will most likely be wrong for many Liouville numbers that are normal to any base $b \geq 2$, whose existence was established in [5]. Theorem 3.2 suggests that there should be equality for some Liouville numbers normal to any base, though, because for generic choices of the $q_{l}$ there is no reason for the resulting Liouville number not to be normal in any base.

REMARK 5.2. Roughly speaking, the approximation functions $\psi_{k, j}(Q)$ introduced in Section 4, related to $\left(\zeta, \zeta^{2}, \ldots, \zeta^{k}\right)$ with $\zeta$ as in Theorem 3.2, all have the maximum possible range, that is $(-1,0)$ for $j=1$ and $((j-k-1) / k j, 1 / k)$ for $2 \leq j \leq k+1$, in any interval $\left(Q_{0}, \infty\right)$. This case was already constructed in $[19$, Corollary 3$]$ for $\zeta \in \mathbb{R}^{k}$ that satisfy the $\mathbb{Q}$-linear independence property, however Theorem 3.2 provides examples for the special case $\underline{\zeta}=\left(\zeta, \zeta^{2}, \ldots, \zeta^{k}\right)$. 
5.2. Specifications. The most obvious way to specify Theorem 3.2 is to put $q_{l}=b^{a_{l}}$ for some fixed integer $b \geq 2$.

COROLlary 5.3. Let $\left(a_{l}\right)_{l \geq 1}$ be a strictly increasing sequence of positive integers with the property

$$
\limsup _{n \rightarrow \infty} \min \left\{\frac{a_{n+1}}{a_{n}}, \frac{a_{n+2}}{a_{n+1}}\right\}=\infty .
$$

Let $b \geq 2$ be an integer and let $\zeta=\sum_{l \geq 1} b^{-a_{l}}$. Then the quantities $\lambda_{k, j}(\zeta), \widehat{\lambda}_{k, j}(\zeta)$ are given as in Theorem 3.2 for any positive integer $k$.

Proof. Apply Theorem 3.2 with $q_{l}:=b^{a_{l}}$ and note that (3.5) follows from (5.7).

The condition (5.7) of Corollary 5.3 is in particular satisfied if

$$
\lim _{n \rightarrow \infty} \frac{a_{n+1}}{a_{n}}=\infty .
$$

which allows to specify Corollary 5.3 further in two ways.

Corollary 5.4. Let $L$ be as in (1.2). Then for any positive integer $k$ and $1 \leq j \leq k+1$ the quantities $\lambda_{k, j}(L), \widehat{\lambda}_{k, j}(L)$ are given as in Theorem 3.2.

Proof. Let $b=10$ and $a_{l}=l$ ! in Corollary 5.3 and observe that (5.8) holds.

Recall the Cantor middle third set is the set of numbers in $[0,1]$ which can be written without the digit 1 in the 3 -adic expansion.

Corollary 5.5. The number $\zeta=2 \cdot \sum_{l \geq 1} 3^{-l !}$ is an element of the Cantor middle third set, whose approximation constants $\lambda_{k, j}(\zeta), \widehat{\lambda}_{k, j}(\zeta)$ are given as in Theorem 3.2 for any positive integer $k$.

Proof. Let $b=3$ and $a_{l}=l$ !, then (5.8) holds and we may apply Corollary 5.3 to $\zeta / 2$. Finally rational transformations do not affect the quantities $\lambda_{k, j}(\zeta), \widehat{\lambda}_{k, j}(\zeta)$.

Recall $\chi_{k, j}$ from (1.8). Theorem 3.2, Corollary 5.5 and the bounds of $\lambda_{k, j}(\zeta)$ from (1.9) suggest the following generalizations of Conjecture 1.1.

Conjecture 5.6 (Weak). Let $k$ be a positive integer and $1 \leq j \leq k+1$. The (individual) spectrum of $\lambda_{k, j}(\zeta)$ among all real $\zeta$ not algebraic of degree $\leq k$ is $\left[\chi_{k, j}, 1 /(j-1)\right]$.

Conjecture 5.7 (Strong). Let $k$ be a positive integer and $1 \leq j \leq k+1$. The (individual) spectrum of $\lambda_{k, j}(\zeta)$ among irrational $\zeta$ in the Cantor set is $\left[\chi_{k, j}, 1 /(j-1)\right]$. 
Recall that the analogue of the conjectures for the uniform constants $\widehat{\lambda}_{k, j}(\zeta)$ with the bounds from (1.10),(1.11) fails heavily due to (1.12).

At this point we want to add that unmentioned there, similar to Corollary 5.5 one can choose numbers in the Cantor middle third set satisfying the results in [19, Section 2] by small rearrangements. A concrete example is changing the base from 2 to 3 and replacing each $\zeta_{j}$ by $2 \zeta_{j}$ in $[19$, Corollary 7].

Eventually, we want to point out that the results in Section 3 have an equivalent interpretation in a linear form problem. Define $w_{k, j}(\zeta)$ resp. $\widehat{w}_{k, j}(\zeta)$ as the supremum of real $\nu$ such that the system

$$
\left|x_{0}\right| \leq X, \quad\left|x_{0}+\zeta x_{1}+\cdots+\zeta^{k} x_{k}\right| \leq X^{-\nu}
$$

has (at least) $j$ linearly independent solutions $\left(x_{0}, \ldots, x_{k}\right) \in \mathbb{Z}^{k+1}$ for arbitrarily large $X$ resp. for all sufficiently large $X$. Then, the identities

$$
w_{k, j}(\zeta)=\frac{1}{\widehat{\lambda}_{k, k+2-j}(\zeta)}, \quad \widehat{w}_{k, j}(\zeta)=\frac{1}{\lambda_{k, k+2-j}(\zeta)}
$$

hold for $1 \leq j \leq k+1$, see [20, (1.24)]. Thus, the constants $w_{k, j}(\zeta), \widehat{w}_{k, j}(\zeta)$ can be readily determined for Liouville numbers $\zeta$ involved in Section 3. Observe that $w_{k, 1}(\zeta)$ resp. $\widehat{w}_{k, 1}(\zeta)$ coincide with the well-studied constants $w_{k}(\zeta)$ resp. $\widehat{w}_{k}(\zeta)$ (at least for $\zeta$ not algebraic of degree $\left.\leq k\right)$ introduced by Mahler ([10, 11]) in 1932.

\section{ACKNOWLEDGEMENTS.}

Thanks to the referee for many structural advices!

\section{REFERENCES}

[1] V. Beresnevich, D. Dickinson and S. Velani, Measure theoretic laws for lim sup sets, Mem. Amer. Math. Soc. 179 (2006), no. 846.

[2] V. Beresnevich, D. Dickinson and S. L. Velani, Diophantine approximation on planar curves and the distribution of rational points with an appendix by R.C. Vaughan, Sums of two squares near perfect squares, Ann. Math. (2) 166 (2007), 367-426.

[3] Y. Bugeaud, On simultaneous rational approximation to a real numbers and its integral powers, Ann. Inst. Fourier (Grenoble) 60 (2010), 2165-2182.

[4] Y. Bugeaud and M. Laurent, Exponents of Diophantine Approximation and Sturmian Continued Fractions, Ann. Inst. Fourier (Grenoble) 55 (2005), 773-804.

[5] Y. Bugeaud, Nombres de Liouville et nombres normaux, C. R. Math. Acad. Sci. Paris, 335 (2002), 117-120.

[6] V. Jarník, Contribution á la théorie des approximations diophantiennes linéaires et homogénes, Czech Math. J. 4(79) (1954), 330-353.

[7] A. Y. Khintchine, Zur metrischen Theorie der diophantischen Approximationen, Math. Z. 24 (1926), 706-714

[8] D. Kleinbock, Extremal subspaces and their submanifolds, Geom. Funct. Anal. 13 (2003), 437-466.

[9] M. Laurent, Simultaneous rational approximation to successive powers of a real number, Indag. Math. (N.S.) 14 (2003), 45-53. 
[10] K. Mahler, Zur Approximation der Exponentialfunktionen und des Logarithmus. I, J. Reine Angew. Math. 166 (1932), 118-136.

[11] K. Mahler, Zur Approximation der Exponentialfunktionen und des Logarithmus. II, J. Reine Angew. Math. 166 (1932), 137-150.

[12] H. Minkowski, Geometrie der Zahlen, Bibliotheca Mathematica Teubneriana, New York-London, 1968.

[13] K.F. Roth, Rational approximations to algebraic numbers, Mathematika 2 (1955), 120

[14] D. Roy, Diophantine approximation in small degree, Number theory, E.Z. Goren and H. Kisilevsky Eds, CRM Proceedings and Lecture Notes, 2004, p. 269-285.

[15] D. Roy, On simultaneous rational approximations to a real number, its square and its cube, Acta Artih. 133 (2008), 185-197.

[16] D. Roy, On Schmidt and Summerer parametric geometry of numbers, arXiv: 1406.3669

[17] D. Roy, Construction of points realizing the regular systems of Wolfgang Schmidt and Leonhard Summerer, arXiv:1406.3669

[18] D. Roy, Spectrum of the exponents of best rational approximation, arXiv:1410.1007.

[19] J. Schleischitz, Diophantine approximation and special Liouville numbers, Commun. Math. 21 (2013), 39-76.

[20] J Schleischitz, Two estimates concerning classical diophantine approximation constants, Publ. Math. Debrecen, 84 (2014), 415-437.

[21] J. Schleischitz, On the spectrum of Diophantine approximation constants, Mathematika, to appear.

[22] J. Schleischitz, Generalizations of a result of Jarnik on simultaneous approximation, arXiv: 1410.6697.

[23] W. M. Schmidt, Norm form equations, Ann. of Math. (2) 96 (1972), 526-551.

[24] W. M. Schmidt and L. Summerer, Parametric geometry of numbers and applications, Acta Arith. 140 (2009), 67-91.

[25] W. M. Schmidt and L. Summerer, Diophantine approximation and parametric geometry of numbers, Monatsh. Math. 169 (2013), 51-104.

[26] W. M. Schmidt and L. Summerer, Simultaneous approximation to three numbers, Mosc. J. Comb. Number Theory 3 (2013), 84-107.

[27] V. G. Sprindẑuk, A proof of Mahler's conjecture on the measure of the set of $S$ numbers, Izv. Akad. Nauk SSSR Ser. Mat. 29 (1965), 379-436. English translation in: Amer. Math. Soc. Transl. 51 (1966), 215-272.

[28] R. C. Vaughan and S. Velani, Diophantine approximation on planar curves: the convergence theory, Invent. Math. 166 (2006), 103-124.

[29] M. Waldschmidt, Recent advances in Diophantine approximation, Number theory, analysis and geometry, Springer, New York, 2012, p. 659-704

J. Schleischitz

Institute of Mathematics

Univ. Nat. Res. Life Sci.

Gregor-Mendel-Straße 33, Vienna, 1180

Austria

E-mail: johannes.schleischitz@boku.ac.at

Received: 4.11.2014.

Revised: 10.12 .2014 . \& 14.1.2015. 